liposomes is specific for structures containing gangliosides or a positive lipid such as stearylamine. The types of binding to these two sorts of liposomes are clearly differentiated, however, since only the membranes which incorporate the sialic groups can compete effectively for virus in mixture with erythrocytes. The weak nonspecific binding of Sendai virus to liposomes containing stearylamine is probably electrostactic since the virions have a net negative charge.

The way in which sialic acid groups are presented to the virus is of prime importance for high affinity binding. For example, very small liposomes containing gangliosides obtained by sonication are less potent inhibitors of viral haemagluttination than are liposomes with a greater total surface area. Second, ganglioside micelles do not compete with erythrocytes for virus. Probably both a threshold surface area and ganglioside density are needed in order that the virus can make a critical number of contracts with the absorbing surface. A somewhat similar interpretation probably explains the finding, made earlier by several groups, that the tightness of binding of myxoviruses to sialoglycoproteins depends critically on the molecular size and is drastically decreased by proteolysis of the receptor glycoproteins. The reason that a large number of contacts are required for firm attachment to receptors containing sialic acid might be explained if the bond energy between receptor groups and viral spike glycoproteins is low.

Whether gangliosides or sialoglycoproteins or both are the naturally important receptors for Sendai virus in cells which support a lytic infection is unknown. Haywood suggests that the probable topography of the cell surface is such that the first viral contacts may be with glycoproteins, after which virions are handed over to gangliosides situated nearer to the bilayer and in a better position to initiate events leading to penetration. Perhaps this process involves the viral neuraminidase in localised reactions which break the initial binding of virions to receptor glycoproteins and allow attachment to internal gangliosides to take place.

\section{New advances in SEM}

\section{from a Correspondent}

At the seventh Annual IIT Research Institute Symposium on Scanning Electron Microscopy in Chicago from April 7 to 11, probably the most significant advance in instrumentation was that reported by $\mathrm{O}$. Wells and $\mathrm{A}$. Broers (IBM Research Laboratories). These workers immersed the specimen in the high field region of the final lens and collected the low energy loss electrons coming from the edge of a highly tilted specimen. They were then able to demonstrate centre/centre resolution of $3 \mathrm{~nm}$ and an edge resolution of $1.5 \mathrm{~nm}$. The instrument used was the $100 \mathrm{Kv} \mathrm{LaB}_{6}$ gun designed by Broers, who thought that the resolution could now only be further improved by using a single crystal of $\mathrm{LaB}_{;}$to replace the finely pointed sintred rod.

M. Isaacson (University of Chicago) presented a frank discussion of the problems associated with specimen preparation for the high resolution scanning transmission electron microscope presently under development in several laboratories. Although the instrumental potential now exists for atomic resolution, specimen contamination and beam damage represent real problems which must be solved before a wide range of biological macromolecules are going to be imaged in these new microscopes. Isaacson and his colleagues considered the answer to these problems lay in the use of LHe II stages, low atomic weight negative stains and computer image processing to remove background noise.

Several speakers "discussed realtime stereo viewing and dimensional reconstruction of stereo pairs. A. Boyde (University College, London) outlined the problems associated with such reconstructions and demonstrated the usefulness of the method in analyses of complex tri-dimensional objects and in interpreting dynamic experiments in the microscope. But the most convincing demonstration of real-time stereo viewing was given by $\mathrm{E}$. Chatfield and colleagues (Ontario Research Foundation). By using a single post lens deflection coil they took a dual signal which was displayed on a colour TV set utilising the red and green guns. The image could then be viewed using red and green separation filters in order to reconstitute the 3D image.

N. Barbi and colleagues (Prairie View, Illinois) gave some data on a windowless energy dispersive X-ray detector in which it was claimed that they are able to get significant signals for $\mathrm{C}, \mathrm{O}$ and $\mathrm{N}$ though problems of contamination and beam collimation still remain. There were several comments on some of the ancillary techniques associated with SEM, including advances in cathodoluminescence, autoradiography and histochemistry. None, however, demonstrated the necessary breakthrough which would make these techniques of any significant use in scanning microscopy. In the biological sciences, J. Nowell (University of California at Davis) gave more details of the injection replication technique for internal cavities of animal tissue and W. Humphreys and colleagues (Uni- versity of Georgia) outlined a useful variation on the critical point drying technique which enables cryofractured specimens to be visualised in the SEM.

Scanning electron microscopy seems to have come of age and there are now more than 25 different models of microscope from which to choose. The IITRI SEM symposia have played no small part in the growth of the subject and it will be of interest to see whether future meetings can continue to contribute to the requirements of scanning electron microscopists and not degenerate into a dreary catalogue of photographs of what can be seen using this instrument.

\section{Activation energies under scrutiny}

from our Chemical Physics Correspondent FOR many years discussions of thermal reaction rates have centred on the activation energies, $E$, of reactions whose rates have followed the Arrhenius expression, $k=A \exp (-E / R T)$ and its more modern variants. Its justification requires reagents to possess the energy $E$ in order to surmount an energy barrier and proceed to reaction. The degrees of freedom holding the energy were considered irrelevant except that their number might influence the factor $A$. It has long been recognised that this assumption was not fully correct but experiments to show the specific influence of particular categories of initial energy have not been easy to devise.

In recent years a number of cases have been studied where the excess energy of the products of an exothermic reaction has resided in specific vibrational excitation. The principle of microscopic reversibility then requires that analagous reactions in the reverse direction should proceed preferentially with the reagents in excited vibrational states. It is just such a process which has been elegantly demonstrated by Douglas, Polanyi and Sloan at Toronto (J. chem. Phys., 59, 6679; 1973).

The reaction chosen was

$$
\mathrm{HCl}+\mathrm{Br} \rightarrow \mathrm{HBr}+\mathrm{Cl}
$$

for which the $\mathrm{HCl}$ reagent could be prepared in excited vibrational states from the reaction

$$
\mathrm{HI}+\mathrm{Cl} \rightarrow \mathrm{HCl}(v=1 \text { to } 4)+\mathrm{I}
$$

Such a stream of vibrationally excited states was crossed with a stream of $\mathrm{Br}$ atoms which was pulsed on and off 15 times a second. The occupancy of the vibrational levels of the $\mathrm{HCl}$ was monitored through the infrared emission spectrum and the change between the on and off periods for the $\mathrm{Br}$ was simultaneously obtained by means of a phase sensitive detector set to $15 \mathrm{~Hz}$. The frequency setting of the spectro- 